\title{
HERMITE SERIES SOLUTIONS OF LINEAR FREDHOLM INTEGRAL EQUATIONS
}

\author{
Salih Yalçınbaş and Müge Aynigül \\ Department of Mathematics, Faculty of Science and Arts, Celal Bayar \\ University, 45047 Muradiye, Manisa, Turkey. \\ syalcin@fef.sdu.edu.tr muge.aynigul@bayar.edu.tr
}

\begin{abstract}
A matrix method for approximately solving linear Fredholm integral equations of the second kind is presented. The solution involves a truncated Hermite series approximation. The method is based on first taking the truncated Hermite series expansions of the functions in equation and then substituting their matrix forms into the equation. Thereby the equation reduces to a matrix equation, which corresponds to a linear system of algebraic equations with unknown Hermite coefficients. In addition, some equations considered by other authors are solved in terms of Hermite polynomials and the results are compared.
\end{abstract}

Keywords- Hermite series, Linear Fredholm integral equations.

\section{INTRODUCTION}

Fredholm integral equations are widely used for modelling and forecasting in almost all areas of science and engineering.

Fredholm integral equations are usually difficult to solve analytically so it is required to obtain an efficient approximate solution. As we know, much work has been done on developing and analyzing numerical methods for solving linear Fredholm integral equations [2-10-11].

One of the orthogonal polynomials is Hermite polynomials $\left\{H_{0}(x), H_{1}(x), H_{2}(x), \ldots, H_{N}(x)\right\}$ which are orthogonal on $(-\infty, \infty)$, so Hermite polynomials have comparison advantages with other orthogonal polynomials.

The subject of the presented paper is to apply the Hermite method for solving linear Fredholm integral equations.

In this paper, we consider the Fredholm integral equations of the second kind

$$
y(x)=f(x)+\lambda \int_{-1}^{1} K(x, t) y(t) d t
$$

where $y(x)$ is the function to be determined. The constant $\lambda$, the kernel function $K(x, t)$ and the function $f(x)$ are given. We assume that the interested domain of the variables is $-1 \leq x, t \leq 1$.

The solution of Eq. (1) is expressed as the truncated Hermite series

$$
y(x)=\sum_{r=0}^{N} a_{r} H_{r}(x)
$$

where $H_{r}(x)$ is the Hermite polynomial of degree $r$ [6], or in the matrix form

$$
[y(x)]=\mathbf{H}_{\mathbf{x}} \mathbf{A}
$$

where 


$$
\begin{aligned}
\mathbf{H}_{\mathbf{x}} & =\left[H_{0}(x) H_{1}(x) H_{2}(x) \ldots H_{N}(x)\right] \\
\mathbf{A} & =\left[a_{0} a_{1} a_{2} \ldots a_{N}\right]^{T}
\end{aligned}
$$

and $a_{r}, r=0,1, \ldots, N$ are coefficients to be determined.

\section{METHOD FOR SOLUTION}

To obtain the solution of Eq. (1) in the form of expression (2) we can first deduce the following matrix approximations corresponding to the Hermite series expansions of the functions $f(x), K(x, t)$ and $y(t)$.

Let the function $f(x)$ be approximated by a truncated Hermite series

$$
f(x)=\sum_{r=0}^{N} f_{r} H_{r}(x) .
$$

Then we can put series (4) in the matrix form

$$
[f(x)]=\mathbf{H}_{\mathbf{x}} \mathbf{F}
$$

where

$$
\mathbf{F}=\left[f_{0} f_{1} \ldots f_{N}\right]^{T} .
$$

We now consider the kernel function $K(x, t)$. If the function $K(x, t)$ can be approximated by double Hermite series of degree $\mathrm{N}$ in both $x$ and $t$ of the form [2,7]

$$
K(x, t)=\sum_{r=0}^{N} \sum_{s=0}^{N} k_{r, s} H_{r}(x) H_{s}(t)
$$

then we can put series (6) in the matrix form

$$
[K(x, t)]=\mathbf{H}_{\mathbf{x}} \mathbf{K H}_{\mathbf{t}}^{\mathbf{T}}
$$

where

$$
\begin{aligned}
\mathbf{H}_{\mathbf{t}} & =\left[H_{0}(t) H_{1}(t) \ldots H_{N}(t)\right] \\
\mathbf{K} & =\left[\begin{array}{cccc}
k_{00} & k_{01} & \cdots & k_{0 N} \\
k_{10} & k_{11} & \cdots & k_{1 N} \\
\vdots & \vdots & \ddots & \vdots \\
k_{N 0} & k_{N 1} & \cdots & k_{N N}
\end{array}\right] .
\end{aligned}
$$

On the other hand, for the unknown function $y(t)$ in integrand, we write from expressions (2) and (3)

$$
[y(t)]=\mathbf{H}_{\mathbf{t}} \mathbf{A} .
$$

Substituting the matrix forms (3), (5), (7) and (8) corresponding to the functions $y(x), f(x), K(x, t)$ and $y(t)$, respectively, into Eq. (1), and then simplifying the result equation, we have the matrix equation

or briefly

$$
\mathbf{A}=\mathbf{F}+\lambda \mathbf{K}\left\{\int_{-1}^{1} \mathbf{H}_{\mathbf{t}}^{\mathbf{T}} \mathbf{H}_{t} d t\right\} \mathbf{A}
$$

where

$$
(\mathbf{I}-\lambda \mathbf{K Q}) \mathbf{A}=\mathbf{F}
$$




$$
\mathbf{Q}=\int_{-1}^{1} \mathbf{H}_{\mathbf{t}}^{\mathrm{T}} \mathbf{H}_{\mathbf{t}} d t=\left[q_{r s}\right], \quad r, s=0,1, \ldots, N
$$

and $\mathbf{I}$ is the unit matrix; the elements of the fixed matrix $\mathbf{Q}$ are similar by [1,2]

$$
q_{r s}=\int_{-1}^{1} H_{r}(t) H_{s}(t) d t .
$$

In Eq. (9), if $D(\lambda)=|\mathbf{I}-\lambda \mathbf{K Q}| \neq 0$ we get

$$
\mathbf{A}=(\mathbf{I}-\lambda \mathbf{K} \mathbf{Q})^{-1} \mathbf{F}, \lambda \neq 0 .
$$

Thus the unknown coefficients $a_{r}, \quad r=0,1, \ldots, N$ are uniquely determined by equation (11) and thereby the integral Eq. (1) has a unique solution. This solution is given by the truncated Hermite series (2).

\section{ACCURACY OF SOLUTION}

We can easily check the accuracy of the method. Since the truncated Hermite series in (2) is an approximate solution of Eq. (1), it must be approximately satisfied this equation.

Then for each $x_{i} \in[-1,1]$

$$
E\left(x_{i}\right)=\left|y\left(x_{i}\right)-f\left(x_{i}\right)-\lambda \int_{-1}^{1} K\left(x_{i}, t\right) y(t) d t\right| \cong 0
$$

or

$$
E\left(x_{i}\right) \leq 10^{-k_{i}} \quad\left(k_{i} \text { is any positive integer }\right)
$$

If

$$
\max \left(10^{-k_{i}}\right)=10^{-k} \quad(k \text { is any positive integer })
$$

is prescribed, then the truncation limit $N$ is increased until the difference $E\left(x_{i}\right)$ at each of the points $x_{i}$ becomes smaller than the prescribed $10^{-k}$.

On the other hand, the error function can be estimated by

$$
E(x)=y(x)-f(x)-\lambda \int_{-1}^{1} K(x, t) y(t) d t[6] .
$$

\section{NUMERICAL ILLUSTRATIONS}

We show the efficiency of the presented method using the following examples.

Example 1. Let us first consider the linear Fredholm integral equation [2,3]

$$
y(x)=(x+1)^{2}+\int_{-1}^{1}\left(x t+x^{2} t^{2}\right) y(t) d t
$$

and seek the solution $y(x)$ in Hermite series

so that

$$
y(x)=\sum_{r=0}^{N} a_{r} H_{r}(x)
$$

$$
f(x)=x^{2}+2 x+1 \quad K(x, t)=\left(x t+x^{2} t^{2}\right) \quad \lambda=1 \quad N=2 .
$$


By using the expansions for the powers $x^{r}$ in terms of the Hermite polynomials $H_{r}(x)[6]$, we easily find the representations

$$
f(x)=x^{2}+2 x+1=\frac{3}{2} H_{0}(x)+H_{1}(x)+\frac{1}{4} H_{2}(x)
$$

and

$$
K(x, t)=\left(x t+x^{2} t^{2}\right)=\frac{1}{4} H_{0}(x) H_{0}(t)+\frac{1}{8} H_{2}(x) H_{0}(t)+\frac{1}{4} H_{1}(x) H_{1}(t)+\frac{1}{8} H_{0}(x) H_{2}(t)+\frac{1}{16} H_{2}(x) H_{2}(t)
$$

and hence, from relations (5) and (7), the matrices

$$
\mathbf{F}=\left[\begin{array}{c}
3 / 2 \\
1 \\
1 / 4
\end{array}\right] \quad, \quad \mathbf{K}=\frac{1}{8}\left[\begin{array}{ccc}
2 & 0 & 1 \\
0 & 2 & 0 \\
1 & 0 & 1 / 2
\end{array}\right]
$$

If we use expression (10) for $r, s=0,1,2$, we obtain the fixed matrix

$$
\mathbf{Q}=\left[\begin{array}{ccc}
2 & 0 & -4 / 3 \\
0 & 8 / 3 & 0 \\
-4 / 3 & 0 & 56 / 15
\end{array}\right]
$$

Next, we substitute these matrices into Eq. (11) and then simplify to obtain

The solution of this equation is

$$
\left[\begin{array}{l}
a_{0} \\
a_{1} \\
a_{2}
\end{array}\right]=\left[\begin{array}{ccc}
14 / 9 & 0 & 2 / 9 \\
0 & 3 & 0 \\
5 / 18 & 0 & 10 / 9
\end{array}\right]\left[\begin{array}{c}
3 / 2 \\
1 \\
1 / 4
\end{array}\right] .
$$

$$
a_{0}=43 / 18 \quad a_{1}=3 \quad a_{2}=25 / 36 .
$$

By substituting the obtained coefficients in (2) the solution of (12) becomes

$$
y(x)=\frac{43}{18} H_{0}(x)+3 H_{1}(x)+\frac{25}{36} H_{2}(x)
$$

or

$$
y(x)=\frac{25}{9} x^{2}+6 x+1
$$

which is the exact solution.

Example 2. We can study the following linear Fredholm integral equation $[5,8]$

$$
y(x)=0.9 x^{2}+0.5 \int_{0}^{1} x^{2} t^{2} y(t) d t
$$

If we use expression (10) for $r, s=0,1,2$, we obtain $\mathbf{Q}$ matrix. Next substitute these matrices in Eq. (11) and then simply obtain

$$
y(x)=x^{2}
$$

which is the exact solution.

Example 3. Let us now consider [10]

$$
y(x)=e^{2 x}-x+\int_{0}^{1} x e^{-2 t} y(t) d t .
$$


The comparison of solutions (for $N=7-10$ ) with exact solution $e^{2 x}$ is given in Table 1 and Figure 1.

Table 1. Comparing the solutions and error analysis which has been found for $N=7-10$ at Example 3 .

\begin{tabular}{|c|c|c|c|c|c|c|c|c|c|c|}
\hline \multirow{3}{*}{$i$} & \multirow{3}{*}{$x_{i}$} & \multicolumn{9}{|c|}{ Present method : Hermite Method } \\
\hline & & \multirow{2}{*}{$\begin{array}{l}\text { Exact Solution } \\
y\left(x_{i}\right)=e^{2 x}\end{array}$} & \multicolumn{2}{|c|}{$N=7$} & \multicolumn{2}{|c|}{$N=8$} & \multicolumn{2}{|c|}{$N=9$} & \multicolumn{2}{|c|}{$N=10$} \\
\hline & & & $y\left(x_{i}\right)$ & $E\left(x_{i}\right)$ & $y\left(x_{i}\right)$ & $E\left(x_{i}\right)$ & $y\left(x_{i}\right)$ & $E\left(x_{i}\right)$ & $y\left(x_{i}\right)$ & $E\left(x_{i}\right)$ \\
\hline 0 & 0 & 1 & 1 & 0 & 1 & 0 & 1 & 0 & 1 & 0 \\
\hline 1 & 0.1 & 1.221402758 & 1.22096 & $4.3 \mathrm{E}-04$ & 1.22148 & $8.3 \mathrm{E}-04$ & 1.22138 & $1.6 \mathrm{E}-05$ & 1.22140 & $2.6 \mathrm{E}-06$ \\
\hline 2 & 0.2 & 1.491824698 & 1.49094 & $8.7 \mathrm{E}-04$ & 1.49199 & $1.6 \mathrm{E}-04$ & 1.49179 & $3.3 \mathrm{E}-05$ & 1.49183 & $5.3 \mathrm{E}-06$ \\
\hline 3 & 0.3 & 1.8221188 & 1.82080 & $1.3 \mathrm{E}-03$ & 1.82237 & $2.5 \mathrm{E}-04$ & 1.82206 & $5.0 \mathrm{E}-05$ & 1.82212 & 8.0 E-06 \\
\hline 4 & 0.4 & 2.225540928 & 2.22378 & $1.7 \mathrm{E}-03$ & 2.22587 & $3.3 \mathrm{E}-04$ & 2.22547 & $6.7 \mathrm{E}-05$ & 2.22555 & $1.0 \mathrm{E}-05$ \\
\hline 5 & 0.5 & 2.718281828 & 2.71606 & $2.2 \mathrm{E}-03$ & 2.71869 & $4.1 \mathrm{E}-04$ & 2.71819 & $8.4 \mathrm{E}-05$ & 2.71829 & $1.3 \mathrm{E}-05$ \\
\hline 6 & 0.6 & 3.320116923 & 3.31736 & $2.7 \mathrm{E}-03$ & 3.32060 & $4.8 \mathrm{E}-04$ & 3.32001 & $1.0 \mathrm{E}-04$ & 3.32013 & $1.55 \mathrm{E}-05$ \\
\hline 7 & 0.7 & 4.055199967 & 4.05169 & $3.5 \mathrm{E}-03$ & 4.05572 & $5.2 \mathrm{E}-04$ & 4.05507 & $1.2 \mathrm{E}-04$ & 4.05521 & $1.7 \mathrm{E}-05$ \\
\hline 8 & 0.8 & 4.953032424 & 4.94823 & $4.7 \mathrm{E}-03$ & 4.95347 & $4.4 \mathrm{E}-04$ & 4.95286 & $1.7 \mathrm{E}-04$ & 4.95304 & $1.6 \mathrm{E}-05$ \\
\hline 9 & 0.9 & 6.049647464 & 6.04230 & $7.3 \mathrm{E}-03$ & 6.04973 & $9.1 \mathrm{E}-05$ & 6.04937 & $2.6 \mathrm{E}-04$ & 6.04965 & $5.2 \mathrm{E}-06$ \\
\hline 10 & 1 & 7.389056099 & 7.37656 & $1.2 \mathrm{E}-02$ & 7.38813 & $9.1 \mathrm{E}-04$ & 7.38854 & $5.1 \mathrm{E}-04$ & 7.38902 & $3.4 \mathrm{E}-05$ \\
\hline
\end{tabular}

Hermite Method

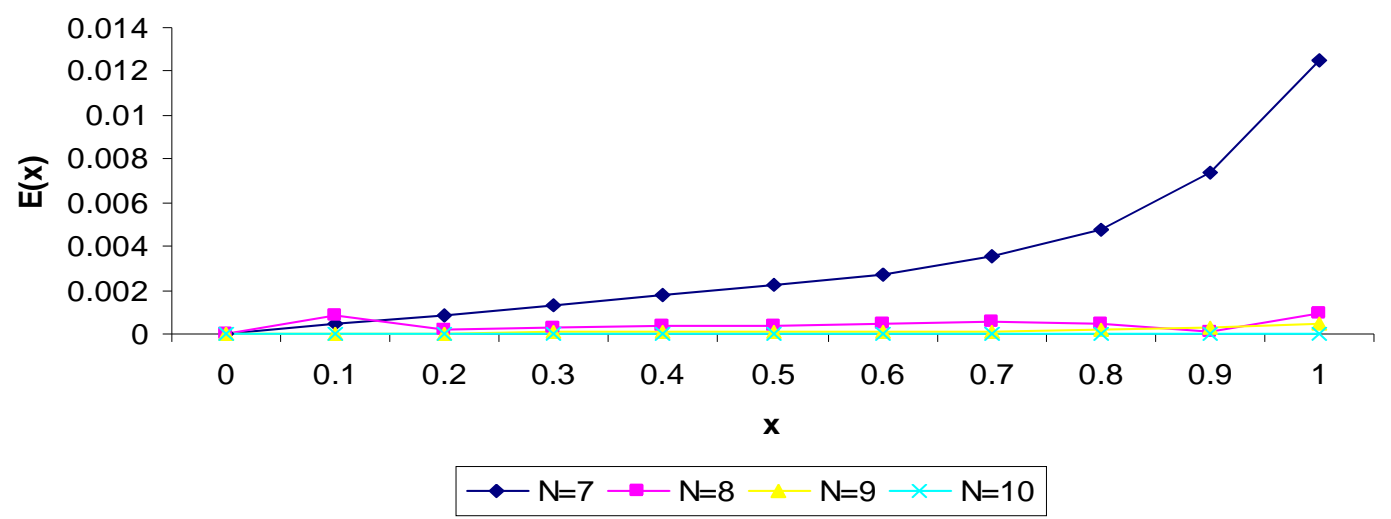

Figure 1. The absolute errors of Example 3 for $N=7-10$.

Example 4. We consider the problem

$$
y(x)=2-e^{x}+\int_{-1}^{1} e^{-t} y(t) d t .
$$

We give numerical analysis for $N=7-10$ in Table 2 and Figure 2.

Example 5. Let us consider the Fredholm integral equation of second kind

$$
y(x)=e^{2 x}-2+\int_{-1}^{1} e^{-2 t} y(t) d t .
$$

The results obtained for $y(x)$ with various $N$ values are presented in Table 3. 
Table 2. Comparing the solutions and error analysis which has been found for $N=7-10$ at Example 4.

\begin{tabular}{|c|c|c|c|c|c|c|c|c|c|c|}
\hline \multirow{3}{*}{$i$} & \multirow{3}{*}{$x_{i}$} & \multicolumn{9}{|c|}{ Present method : Hermite Method } \\
\hline & & \multirow{2}{*}{$\begin{array}{l}\text { Exact Solution } \\
y\left(x_{i}\right)=e^{x}\end{array}$} & \multicolumn{2}{|c|}{$N=7$} & \multicolumn{2}{|c|}{$N=8$} & \multicolumn{2}{|c|}{$N=9$} & \multicolumn{2}{|c|}{$N=10$} \\
\hline & & & $y\left(x_{i}\right)$ & $E\left(x_{i}\right)$ & $y\left(x_{i}\right)$ & $E\left(x_{i}\right)$ & $y\left(x_{i}\right)$ & $E\left(x_{i}\right)$ & $y\left(x_{i}\right)$ & $E\left(x_{i}\right)$ \\
\hline 0 & -1 & 0.367879441 & 0.36786 & $1.1 \mathrm{E}-05$ & 0.36788 & $1.7 \mathrm{E}-06$ & 0.36787 & $1.5 \mathrm{E}-07$ & 0.36787 & $1.7 \mathrm{E}-08$ \\
\hline 1 & -0.8 & 0.449328964 & 0.44933 & $7.1 \mathrm{E}-06$ & 0.44932 & $4.1 \mathrm{E}-07$ & 0.44932 & $7.4 \mathrm{E}-08$ & 0.44932 & 3.9 E-09 \\
\hline 2 & -0.6 & 0.548811636 & 0.54882 & $1.0 \mathrm{E}-05$ & 0.54881 & $7.2 \mathrm{E}-07$ & 0.54881 & $1.0 \mathrm{E}-07$ & 0.54881 & $5.8 \mathrm{E}-09$ \\
\hline 3 & -0.4 & 0.670320046 & 0.67033 & $1.1 \mathrm{E}-05$ & 0.67031 & 7.4 E-07 & 0.67032 & 1.0 E-07 & 0.67032 & 5.9 E-09 \\
\hline 4 & -0.2 & 0.818730753 & 0.81874 & $1.1 \mathrm{E}-05$ & 0.81873 & 7.4 E-07 & 0.81873 & $1.0 \mathrm{E}-07$ & 0.81873 & $5.9 \mathrm{E}-09$ \\
\hline 5 & 0 & 1 & 1 & $1.1 \mathrm{E}-05$ & 0.99999 & $7.4 \mathrm{E}-07$ & 1 & $1.0 \mathrm{E}-07$ & 0.99999 & $5.9 \mathrm{E}-09$ \\
\hline 6 & 0.2 & 1.221402758 & 1.22141 & $1.1 \mathrm{E}-05$ & 1.22140 & $7.4 \mathrm{E}-07$ & 1.22140 & $1.0 \mathrm{E}-07$ & 1.22140 & $6 \mathrm{E}-09$ \\
\hline 7 & 0.4 & 1.491824698 & 1.49183 & $1.1 \mathrm{E}-05$ & 1.49182 & $7.5 \mathrm{E}-07$ & 1.49182 & 1.0 E-07 & 1.49182 & $6 \mathrm{E}-09$ \\
\hline 8 & 0.6 & 1.8221188 & 1.82212 & $1.1 \mathrm{E}-05$ & 1.82211 & $7.7 \mathrm{E}-07$ & 1.82211 & $1.0 \mathrm{E}-07$ & 1.82211 & $6 \mathrm{E}-09$ \\
\hline 9 & 0.8 & 2.225540928 & 2.22554 & $6.4 \mathrm{E}-06$ & 2.22553 & $1.1 \mathrm{E}-06$ & 2.22554 & $7.1 \mathrm{E}-08$ & 2.22554 & $8 \mathrm{E}-09$ \\
\hline 10 & 1 & 2.718281828 & 2.71826 & 1.6 E-05 & 2.71827 & $3.8 \mathrm{E}-06$ & 2.71828 & $2 \mathrm{E}-07$ & 2.71828 & 3.3 E-08 \\
\hline
\end{tabular}

Table 3. Exact, approximate solution and error analysis of Example 5 for the $x$ values

\begin{tabular}{|c|c|c|c|c|c|c|c|c|}
\hline \multirow{3}{*}{$i$} & \multirow{3}{*}{$x_{i}$} & \multicolumn{7}{|c|}{ Present method : Hermite Method } \\
\hline & & Exact Solution & \multicolumn{2}{|c|}{$N=8$} & \multicolumn{2}{|c|}{$N=9$} & \multicolumn{2}{|c|}{$N=10$} \\
\hline & & $y\left(x_{i}\right)=e^{2 x}$ & $y\left(x_{i}\right)$ & $E\left(x_{i}\right)$ & $y\left(x_{i}\right)$ & $E\left(x_{i}\right)$ & $y\left(x_{i}\right)$ & $E\left(x_{i}\right)$ \\
\hline 0 & -1 & 0.1353352832 & 0.13596 & $6.2 \mathrm{E}-04$ & 0.13520 & $1.3 \mathrm{E}-04$ & 0.13536 & $2.6 \mathrm{E}-05$ \\
\hline 1 & -0.9 & 0.1652988882 & 0.16521 & $8.1 \mathrm{E}-05$ & 0.16532 & $2.4 \mathrm{E}-05$ & 0.16529 & $3.4 \mathrm{E}-06$ \\
\hline 2 & -0.8 & 0.2018965180 & 0.20151 & $3.8 \mathrm{E}-04$ & 0.20197 & $8.2 \mathrm{E}-05$ & 0.20188 & $1.3 \mathrm{E}-05$ \\
\hline 3 & -0.7 & 0.2465969639 & 0.24610 & $4.9 \mathrm{E}-04$ & 0.24669 & $1.0 \mathrm{E}-04$ & 0.24658 & $1.6 \mathrm{E}-05$ \\
\hline 4 & -0.6 & 0.3011942119 & 0.30066 & $5.3 \mathrm{E}-04$ & 0.30130 & $1.0 \mathrm{E}-04$ & 0.30117 & $1.7 \mathrm{E}-05$ \\
\hline 5 & -0.5 & 0.3678794412 & 0.36733 & $5.4 \mathrm{E}-04$ & 0.36798 & $1.1 \mathrm{E}-04$ & 0.36786 & $1.7 \mathrm{E}-05$ \\
\hline 6 & -0.4 & 0.4493289641 & 0.44878 & 5.4 E-04 & 0.44943 & $1.1 \mathrm{E}-04$ & 0.44931 & $1.7 \mathrm{E}-05$ \\
\hline 7 & -0.3 & 0.5488116361 & 0.54826 & $5.4 \mathrm{E}-04$ & 0.54892 & $1.1 \mathrm{E}-04$ & 0.54879 & $1.7 \mathrm{E}-05$ \\
\hline 8 & -0.2 & 0.670320046 & 0.66977 & $5.4 \mathrm{E}-04$ & 0.67042 & $1.1 \mathrm{E}-04$ & 0.67030 & $1.7 \mathrm{E}-05$ \\
\hline 9 & -0.1 & 0.8187307531 & 0.81818 & 5.4 E-04 & 0.81884 & $1.1 \mathrm{E}-04$ & 0.81871 & $1.7 \mathrm{E}-05$ \\
\hline 10 & 0 & 1 & 0.99945 & 5.4 E-04 & 1.00010 & $1.1 \mathrm{E}-04$ & 0.99998 & 1.7 E-05 \\
\hline 11 & 0.1 & 1.221402758 & 1.22085 & $5.4 \mathrm{E}-04$ & 1.22151 & $1.1 \mathrm{E}-04$ & 1.22138 & $1.7 \mathrm{E}-05$ \\
\hline 12 & 0.2 & 1.491824698 & 1.49128 & 5.4 E-04 & 1.49193 & $1.1 \mathrm{E}-04$ & 1.49180 & $1.7 \mathrm{E}-05$ \\
\hline 13 & 0.3 & 1.8221188 & 1.82157 & $5.4 \mathrm{E}-04$ & 1.82222 & $1.1 \mathrm{E}-04$ & 1.82210 & $1.7 \mathrm{E}-05$ \\
\hline 14 & 0.4 & 2.225540928 & 2.22499 & $5.4 \mathrm{E}-04$ & 2.22565 & $1.1 \mathrm{E}-04$ & 2.22552 & $1.7 \mathrm{E}-05$ \\
\hline 15 & 0.5 & 2.718281828 & 2.71773 & $5.4 \mathrm{E}-04$ & 2.71839 & $1.1 \mathrm{E}-04$ & 2.71826 & $1.7 \mathrm{E}-05$ \\
\hline 16 & 0.6 & 3.320116923 & 3.31955 & $5.5 \mathrm{E}-04$ & 3.32022 & $1.1 \mathrm{E}-04$ & 3.32009 & $1.8 \mathrm{E}-05$ \\
\hline 17 & 0.7 & 4.055199967 & 4.05459 & $6.1 \mathrm{E}-04$ & 4.05530 & $1.0 \mathrm{E}-04$ & 4.05518 & $1.8 \mathrm{E}-05$ \\
\hline 18 & 0.8 & 4.953032424 & 4.9522 & $7.6 \mathrm{E}-04$ & 4.95310 & $7.3 \mathrm{E}-05$ & 4.95300 & $2.2 \mathrm{E}-05$ \\
\hline 19 & 0.9 & 6.049647464 & 6.04844 & $1.2 \mathrm{E}-03$ & 6.04963 & 8.0 E-06 & 6.04961 & $3.6 \mathrm{E}-05$ \\
\hline 20 & 1 & 7.389056099 & 7.38675 & $2.3 \mathrm{E}-03$ & 7.38882 & $2.3 \mathrm{E}-04$ & 7.38897 & $7.8 \mathrm{E}-05$ \\
\hline
\end{tabular}




\section{Hermite Method}

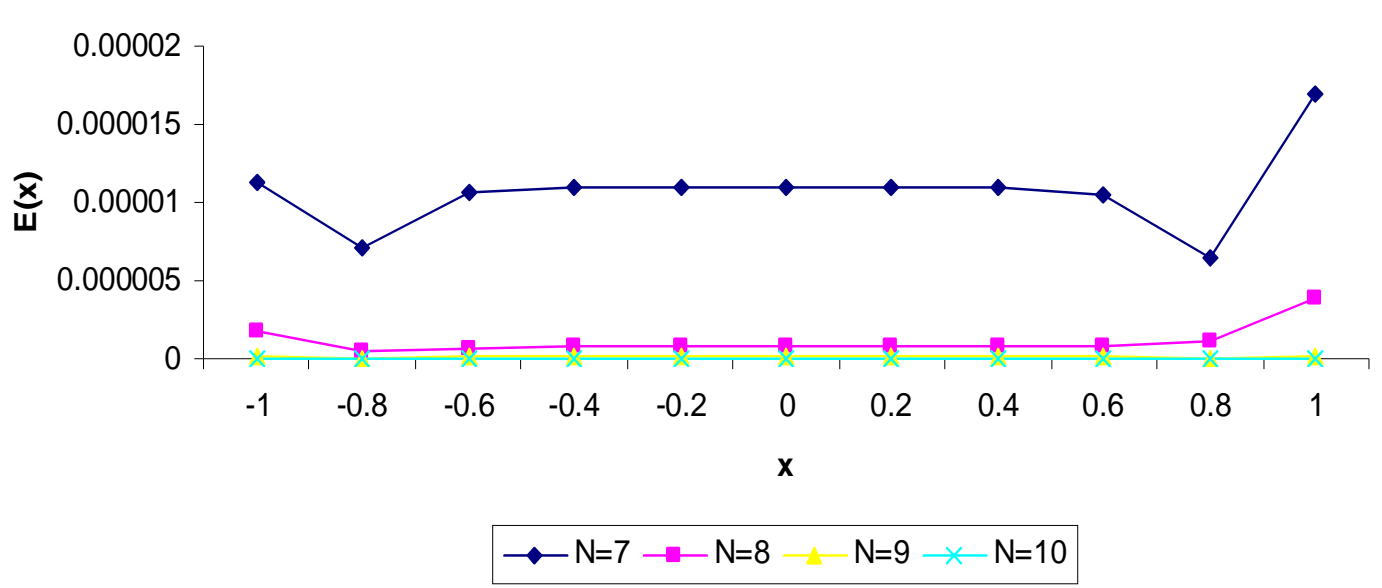

Figure 2. The absolute errors of Example 4 for $N=7-10$.

Example 6. Consider the following the integral equation [12]

$$
y(x)=\int_{0}^{1}(x+1) y(t) d t+e^{x}+(1-e) x-1 .
$$

We give numerical analysis for various $N$ values in Table 4 and Figure 3.

\section{Hermite Method}

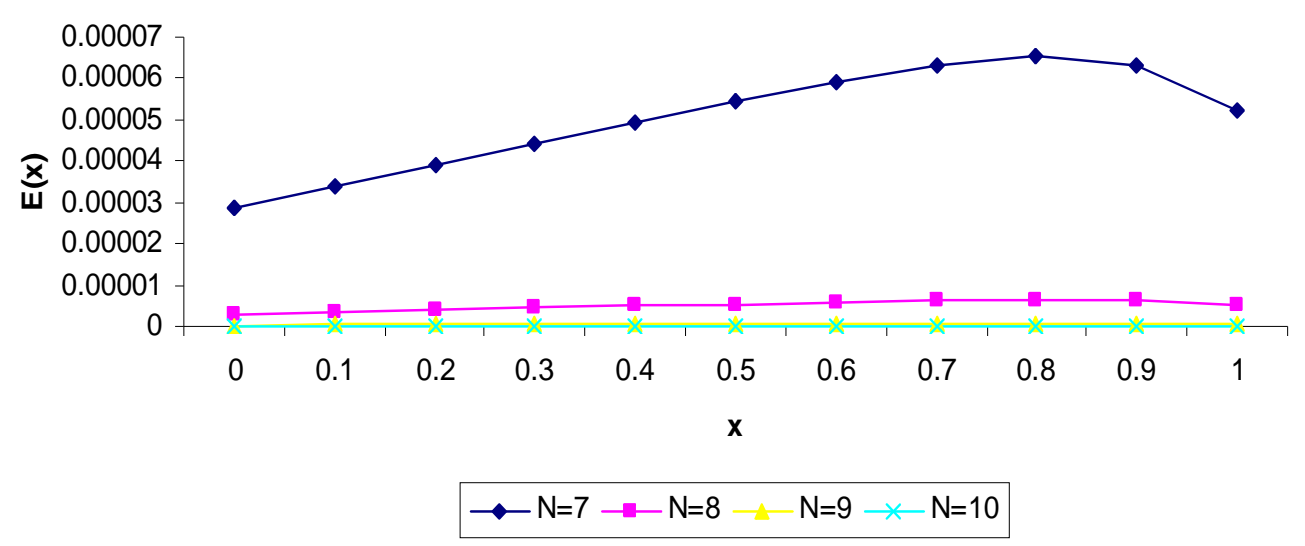

Figure 3. The absolute errors of the solutions by using the present method for different values of $N$. 
Table 4. Comparing the solutions and error analysis which has been found for $N=7-10$ at Example 6.

\begin{tabular}{|c|c|c|c|c|c|c|c|c|c|c|}
\hline \multirow{3}{*}{$i$} & \multirow{3}{*}{$x_{i}$} & \multicolumn{9}{|c|}{ Present method : Hermite Method } \\
\hline & & \multirow{2}{*}{$\begin{array}{l}\text { Exact Solution } \\
y\left(x_{i}\right)=e^{x}\end{array}$} & \multicolumn{2}{|c|}{$N=7$} & \multicolumn{2}{|c|}{$N=8$} & \multicolumn{2}{|c|}{$N=9$} & \multicolumn{2}{|c|}{$N=10$} \\
\hline & & & $y\left(x_{i}\right)$ & $E\left(x_{i}\right)$ & $y\left(x_{i}\right)$ & $E\left(x_{i}\right)$ & $y\left(x_{i}\right)$ & $E\left(x_{i}\right)$ & $y\left(x_{i}\right)$ & $E\left(x_{i}\right)$ \\
\hline 0 & 0 & 1 & 1 & $2.8 \mathrm{E}-05$ & 1 & $2.8 \mathrm{E}-06$ & 1 & $2.6 \mathrm{E}-07$ & 1 & $2.2 \mathrm{E}-08$ \\
\hline 1 & 0.1 & 1.105170918 & 1.10520 & $3.3 \mathrm{E}-05$ & 1.10517 & $3.3 \mathrm{E}-06$ & 1.10517 & $3.0 \mathrm{E}-07$ & 1.10517 & $2.6 \mathrm{E}-08$ \\
\hline 2 & 0.2 & 1.221402758 & 1.22144 & $3.9 \mathrm{E}-05$ & 1.22140 & $3.8 \mathrm{E}-06$ & 1.22140 & $3.5 \mathrm{E}-07$ & \begin{tabular}{|l|}
1.22140 \\
\end{tabular} & $2.9 \mathrm{E}-08$ \\
\hline 3 & 0.3 & 1.349858808 & 1.34990 & $4.4 \mathrm{E}-05$ & 1.34986 & $4.4 \mathrm{E}-06$ & 1.34985 & -07 & 1.34985 & $3.3 \mathrm{E}-08$ \\
\hline 4 & 0.4 & 1.491824698 & 1.49187 & $4.9 \mathrm{E}-05$ & 1.49182 & $4.9 \mathrm{E}-06$ & 1.49182 & $4.4 \mathrm{E}-07$ & 1.49182 & $3.7 \mathrm{E}-08$ \\
\hline 5 & 0.5 & 1.648721271 & 1.64877 & 5.4 E-05 & 1.64872 & $5.4 \mathrm{E}-06$ & 1.64872 & $4.9 \mathrm{E}-07$ & 1.64872 & $4.1 \mathrm{E}-08$ \\
\hline 6 & 0.6 & 1.8221188 & 1.82217 & 5.9 E-05 & 1.82212 & $5.9 \mathrm{E}-06$ & 1.82211 & 5.3 E-07 & 1.82211 & $4.5 \mathrm{E}-08$ \\
\hline 7 & 0.7 & 2.013752707 & 2.01381 & $6.3 \mathrm{E}-05$ & 2.01375 & $6.3 \mathrm{E}-06$ & 2.01375 & $5.7 \mathrm{E}-07$ & 2.01375 & $4.9 \mathrm{E}-08$ \\
\hline 8 & 0.8 & 2.225540928 & 2.22560 & $6.5 \mathrm{E}-05$ & 2.22554 & $6.5 \mathrm{E}-06$ & 2.22554 & $6 \mathrm{E}-07$ & \begin{tabular}{|l|}
2.22554 \\
\end{tabular} & $5.1 \mathrm{E}-08$ \\
\hline 9 & 0.9 & 2.459603111 & 2.45966 & $6.3 \mathrm{E}-05$ & 2.45960 & $6.3 \mathrm{E}-06$ & 2.45960 & 5.7 E-07 & 2.45960 & $4.8 \mathrm{E}-08$ \\
\hline 10 & 1 & 2.718281828 & 2.71833 & $5.2 \mathrm{E}-05$ & 2.71828 & $4.9 \mathrm{E}-06$ & 2.71828 & $4.2 \mathrm{E}-07$ & \begin{tabular}{|l|}
2.71828 \\
\end{tabular} & $3.3 \mathrm{E}-08$ \\
\hline
\end{tabular}

Example 7. Our last example is the integral equation [4]

$$
y(x)=e^{2 x+\frac{1}{3}}-\frac{1}{3} \int_{0}^{1} e^{2 x-\frac{5}{3} t} y(t) d t .
$$

We give numerical analysis for various $N$ values in Table 5 and Figure 4.

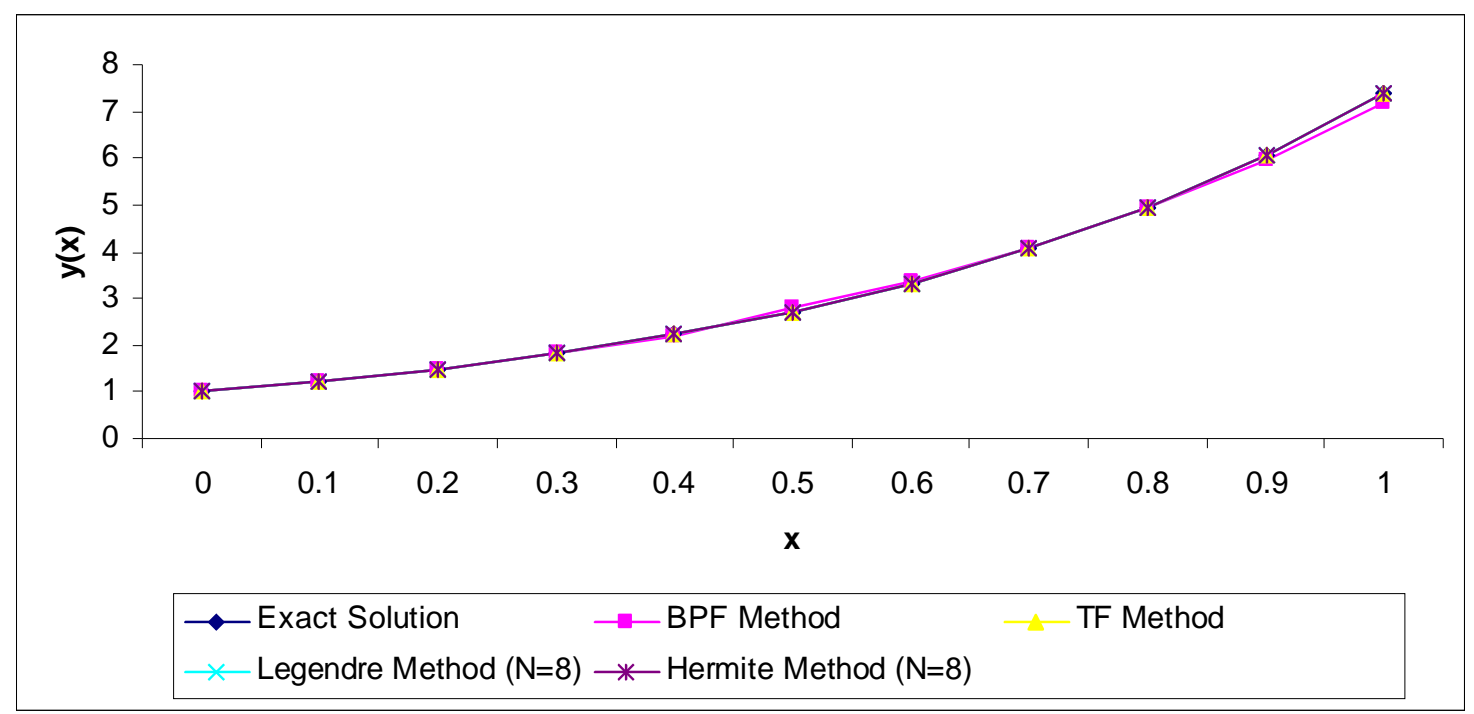

Figure 4. The graphics of the Exact Solution, BPF method, TF Method, Legendre Method and Present method calculated for the values of the interval $[0,1]$. 
Table 5. Error analysis of Example 7 for the $x$ values and comparison of present method, exact and the methods in $[4](N=6,7,8)$.

\begin{tabular}{|c|c|c|c|c|c|c|c|c|}
\hline \multirow[b]{3}{*}{$i$} & \multirow[b]{3}{*}{$x_{i}$} & \multicolumn{7}{|c|}{ Present method :Hermite Method } \\
\hline & & \multirow{2}{*}{$\begin{array}{c}\text { Exact } \\
\text { Solution } \\
y\left(x_{i}\right)=e^{2 x}\end{array}$} & \multicolumn{2}{|c|}{$N=6$} & \multicolumn{2}{|c|}{$N=7$} & \multicolumn{2}{|c|}{$N=8$} \\
\hline & & & $y\left(x_{i}\right)$ & $E\left(x_{i}\right)$ & $y\left(x_{i}\right)$ & $E\left(x_{i}\right)$ & $y\left(x_{i}\right)$ & $E\left(x_{i}\right)$ \\
\hline 0 & 0 & 1 & 0.99915 & 8.4 E-04 & 1.00025 & $2.5 \mathrm{E}-04$ & 0.99997 & $2.6 \mathrm{E}-05$ \\
\hline 1 & 0.1 & 1.2214028 & 1.22037 & $1.0 \mathrm{E}-03$ & 1.22171 & $3.1 \mathrm{E}-04$ & 1.22137 & 3.1 E-05 \\
\hline 2 & 0.2 & 1.4918248 & 1.49056 & $1.2 \mathrm{E}-03$ & 1.49220 & 3.7 E-04 & 1.49178 & 3.9 E-05 \\
\hline 3 & 0.3 & 1.8221188 & 1.82057 & $1.5 \mathrm{E}-03$ & 1.82257 & $4.5 \mathrm{E}-04$ & 1.82207 & 4.7 E-05 \\
\hline 4 & 0.4 & 2.2255408 & 2.22361 & $1.9 \mathrm{E}-03$ & 2.22609 & $5.5 \mathrm{E}-04$ & 2.22548 & $5.8 \mathrm{E}-05$ \\
\hline 5 & 0.5 & 2.7182818 & 2.71576 & $2.5 \mathrm{E}-03$ & 2.71894 & 6.5 E-04 & 2.71820 & 7.4 E-05 \\
\hline 6 & 0.6 & 3.3201163 & 3.31648 & $3.6 \mathrm{E}-03$ & 3.32083 & $7.1 \mathrm{E}-04$ & 3.32001 & $1.0 \mathrm{E}-04$ \\
\hline 7 & 0.7 & 4.0551997 & 4.0492 & $5.9 \mathrm{E}-03$ & 4.05579 & $5.9 \mathrm{E}-04$ & 4.05502 & 1.7 E-04 \\
\hline 8 & 0.8 & 4.9530324 & 4.94224 & $1.1 \mathrm{E}-02$ & 4.95299 & $3.9 \mathrm{E}-05$ & 4.95267 & $3.5 \mathrm{E}-04$ \\
\hline 9 & 0.9 & 6.0496474 & 6.02901 & $2.1 \mathrm{E}-02$ & 6.04777 & $1.8 \mathrm{E}-03$ & 6.04882 & 8.2 E-04 \\
\hline 10 & 1 & 7.3890569 & 7.34935 & $3.9 \mathrm{E}-02$ & 7.38281 & $6.2 \mathrm{E}-03$ & 7.38710 & $1.9 \mathrm{E}-03$ \\
\hline
\end{tabular}

\begin{tabular}{|c|c|c|c|c|}
\hline \multirow{2}{*}{$i$} & \multirow{2}{*}{$x_{i}$} & $\begin{array}{c}\text { Legendre } \\
\text { in [14] }\end{array}$ & $\begin{array}{c}\text { BPF Method in [4] } \\
\text { (Block Pulse Fnc.) }\end{array}$ & $\begin{array}{c}\text { TF Method in } \\
\text { [4] } \\
\text { (Triangular } \\
\text { Fnc.) }\end{array}$ \\
\cline { 3 - 5 } & $N=8$ & \multicolumn{2}{|c|}{$m=32$} \\
\hline 0 & 0 & 0.99999 & 1.031832 & 0.999844 \\
\hline 1 & 0.1 & 1.22137 & 1.244627 & 1.221598 \\
\hline 2 & 0.2 & 1.49180 & 1.501307 & 1.492294 \\
\hline 3 & 0.3 & 1.82210 & 1.810922 & 1.822684 \\
\hline 4 & 0.4 & 2.22550 & 2.184388 & 2.225880 \\
\hline 5 & 0.5 & 2.71821 & 2.804810 & 2.717857 \\
\hline 6 & 0.6 & 3.32001 & 3.383247 & 3.320648 \\
\hline 7 & 0.7 & 4.05502 & 4.080975 & 4.056474 \\
\hline 8 & 0.8 & 4.95266 & 4.922595 & 4.954570 \\
\hline 9 & 0.9 & 6.04882 & 5.937783 & 6.050568 \\
\hline 10 & 1 & 7.38710 & 7.162334 & 7.387901 \\
\hline
\end{tabular}

\section{CONCLUSIONS AND DISCUSSIONS}

In this paper, the usefulness of the method presented for the approximate solution of Fredholm integral equation (1) is demonstrated. To show the accuracy of the method, five integral equations are chosen. A considerable advantage of the method is that the solution is expressed as a truncated Hermite series. This means that, after calculation of the Hermite coefficients, the solution $y(x)$ can be easily evaluated for arbitrary values of $x$ at low computation effort. If the functions $f(x)$ and $K(x, t)$ can be expanded to the Hermite series in $-1 \leq x, t \leq 1$, then there exists the solution $y(x)$; otherwise, the method cannot be used in. On the other hand, it would appear that our method shows to best advantage when the known functions $f(x)$ and $K(x, t)$ have Taylor series about the origin which converge rapidly. To get the best approximating solution of the equation, we must take more terms from the Hermite expansions of functions, 
especially when they converge slowly. Briefly, for computational efficiency, the truncation limit $N$ must be chosen sufficiently large.

\section{REFERENCES}

1. L. Fox, I. Parker, Chebyshev polynomials in Numerical Analysis, Clarendon Press, Oxford, 1968.

2. M. Sezer, S. Dogan, Chebyshev series solutions of Fredholm integral equations, Int. J. Math. Educ. Sci. Technol., 27, 5, 649-657, 1996.

3. R. P. Kanwal, and K. C. Liu, A Taylor expansion approach for solving integral equations, Int. J. Math. Educ. Sci. Technol., 20, 411-414, 1989.

4. E. Babolian, H.R. Marzban, M. Salmani, Using triangular orthogonal functions for solving Fredholm integral equations of the second kind, App. Math. and Comput., 201, 452-464, 2008.

5. R. Farnoosh, M. Ebrahimi, Monte Carlo method for solving Fredholm integral equations of the second kind, App. Math. and Comput., 195, 309-315, 2008.

6. S. Yalçınbaş, M. Sezer, H.H. Sorkun, Legendre polynomial solutions of high-order linear Fredholm integro-differential equations, App. Math. and Comput., 210, 2, 334349, 2009.

7. N. K. Basu, SIAM J. Numer. Anal., 10, 496-505, 1973.

8. K. Maleknejad, M. Karami, Using the WPG method for solving integral equations of the second kind, App. Math. and Comput., 166, 123-130, 2005.

9. K. Maleknejad, Y. Mahmoudi, Numerical solution of linear Fredholm integral equation by using hybrid Taylor and Block-Pulse functions, App. Math. and Comput., 149, 799-806, 2004

10. S. Yalçınbaş, M. Aynigül, T. Akkaya, Legendre series solutions of Fredholm integral equations, Math. and Comput. App., 15, 3, 371-381, 2010.

11. S. Yalçınbaş, T. Akkaya, M. Aynigül, , Laguerre series solutions of Fredholm integral equations, Erciyes University Journal of Science and Technology., 26, 2, 131$142,2010$. 